\title{
LA SEGURIDAD INTERNACIONAL: VINO VIEJO EN BOTELLAS NUEVAS*
}

\author{
International Security: Old Wine in New Bottles
}

\section{ARTURO SOTOMAYOR VELÁZQUEZ}

Centro de Investigación y Docencia Económicas (CIDE)

\begin{abstract}
RESUMEN
La seguridad internacional es un concepto complejo y contestado, cargado de valores y juicios. Por siglos, el concepto fue asociado al estudio de la guerra, la estrategia militar y otras formas alternativas al uso de la fuerza. Mientras pocos disputan este enfoque de la seguridad, otros consideran necesario extender y ampliar su significado para incluir otros valores e intereses. Sugieren incluir temas ambientales, nutrición y protección de derechos humanos. No obstante, ¿dónde deben demarcarse los límites de la seguridad internacional?, ¿qué debe ser excluido e incluido?, ¿la seguridad internacional ha cambiado de tal forma que requerimos una reconceptualización? Este artículo argumenta que en la era de la post-guerra fría la expansión del concepto de seguridad internacional ha incluido tanto en su definición que el proceso de adquisición de conocimiento se ha vuelto fútil. Aún más, se exageran los procesos de cambio internacional en tanto que se olvidan las constantes internacionales en materia de estrategia militar.
\end{abstract}

Palabras clave: seguridad internacional, seguridad humana, análisis conceptual, estrategia militar, post-guerra fría.

\begin{abstract}
International security is a complex and contested concept, heavily laden with values and judgments. For centuries, however, it was linked to the study of war, military strategy and alternatives to the use of force as an instrument of policy. While few would be likely to dispute these examples as issues of security, many would now extend its meaning to other values and interests. They would apply the term to environmental damage, hunger, and protection of human rights. But where do we draw the line in studying international security? What should be included or excluded? Has international security changed in such a way that we now need to re-define the concept? This article argues that in today's post-Cold War world, we seem to have included so much in our definition of security that that we have posed the problem in ways that impede our quest for knowledge. Furthermore, international security, traditionally defined, has suffered few changes since the collapse of the Cold War; thus, there seems to have been more continuity than global change.
\end{abstract}

Key words: international security, human security, conceptual analysis, military strategy, post-cold war.

* El autor desea expresar su agradecimiento a los participantes en el seminario internacional "The Changing International Security Agenda and its Implications for North America", llevado a cabo en la Universidad de las Américas-Puebla, octubre 26-29, 2005. Asimismo, se agradecen las observaciones hechas por Lorena Ruano, Covadonga Meseguer y los dos dictaminadores anónimos de este artículo. Sus comentarios nutrieron este ensayo, aunque por limitaciones de espacio el autor estuvo impedido de introducir todas las sugerencias hechas. Igualmente, se agradece la asistencia de investigación y editorial de Adriana Crespo Tenorio. 


\section{INTRODUCCIÓN}

La idea de que el futuro de la seguridad internacional será muy diferente del pasado reciente se ha vuelto ya una creencia común. Las nuevas tecnologías, el fin de la Guerra Fría y la guerra contra el terrorismo parecen presagiar la llegada de una revolución en asuntos militares con consecuencias potencialmente importantes para la seguridad internacional. De tal forma, de frente a una realidad cambiante, se sugiere que el concepto de seguridad internacional debe igualmente modificarse y adaptarse al nuevo contexto internacional. Por ejemplo, autores como Ann Tickner y Kenn Booth afirman que una ampliación y extensión del concepto de la seguridad ayudará a enfrentar mejor las amenazas y proponer mejores alternativas para garantizar la precaria paz internacional (Tickner, 1995; Booth, 1991). Así pues, si el mundo ha cambiado, ¿por qué no ha de cambiar el concepto de seguridad internacional?

Ciertamente, el cambio es inevitable; no obstante, la realidad internacional, por mutante que sea, siempre conserva rasgos de continuidad. El debate actual sobre la ampliación del concepto de seguridad internacional sistemáticamente exagera los factores de cambio y parece ignorar las constantes históricas. De tal forma, en este ensayo se plantea el siguiente argumento: el arte de la guerra y los instrumentos para garantizar la seguridad internacional han sufrido menos cambios de lo que se suele admitir. Por tanto, el debate sobre la reforma y expansión del concepto de seguridad internacional parecen prematuras porque son las continuidades, más que los cambios, los que dictan las necesidades estratégicas y de seguridad de la mayoría de los Estados. La visión ortodoxa y tradicional sobre seguridad internacional, enfocada en asuntos militares y estratégicos, sigue siendo relevante y no debe ser desplazada por enfoques más normativos o liberales.

Para efectos de este ensayo, se definirá la seguridad internacional como una subdisciplina que forma parte del estudio de las relaciones internacionales y que atiende, esencialmente, a lo referente a la amenaza, uso y control de la fuerza militar. El concepto explora las condiciones que posibilitan el uso de fuerza, los efectos que ésta tiene sobre los individuos, la sociedad y el Estado, así como las políticas disponibles para preparar, prevenir o abrazar la guerra (Walt, 1991: 212). Así planteada, esta definición excluye y está en oposición a una perspectiva liberal sobre la seguridad internacional, según la cual se deben incorporar los actores no estatales y los temas sociales, económicos y ambientales, con el objetivo de atender los asuntos no militares (Booth, 1991; Buzan, Waever y DeWilde, 1998; Martin, 1993; Tickner, 1995).

Este texto está dividido en dos apartados. En la primera sección se presentan y sintetizan los argumentos desarrollados por quienes están a favor de ampliar el concepto de seguridad internacional, introduciendo nuevas agendas y temas que distan de los asuntos militares y de estrategia. Se hará especial mención al esfuerzo reciente por elaborar un concepto holístico y más afín a la empresa liberal, tal como la seguridad humana. La segunda parte de este ensayo presenta una crítica conceptual a los argumentos favorables a la expansión. Dicha crítica está basada en tres tipos de reservas. Dos de ellas son de carácter empírico y se refieren al estado actual que guarda la seguridad internacional y los asuntos militares. 
La última es de tipo metodológico y está relacionada con los problemas que plantea la forma de operacionalizar conceptos amplios, tales como el de la seguridad humana.

\section{EL RECLAMO “LIBERAL” POR EL CAMBIO: UN CONCEPTO DE SEGURIDAD AMPLIO Y EXTENSO}

Los intentos por ampliar el concepto de seguridad internacional ciertamente no son nuevos. La tesis sobre la primacía de la seguridad del individuo por encima de la del Estado era un viejo reclamo del pensamiento europeo de mediados del siglo diecisiete. Fueron la Revolución francesa y las guerras napoleónicas las que terminaron por otorgarle a los temas militares y diplomáticos primacía conceptual (Rothchild, 1995).

Más recientemente, la agenda internacional de la década de los setenta, con su énfasis en el Nuevo Orden Internacional, intentaba ya darle mayor importancia a los temas vinculados con el desarrollo social por encima de los militares. En ese entonces, sin embargo, lo que hoy es considerado como "seguridades económica y política" eran simple y llanamente denominados como estado de bienestar y derechos humanos, formando parte de lo que en aquella época se denominaba como "la agenda de desarrollo internacional". ${ }^{1}$ Igualmente, el Reporte Brandt de la propia ONU, dado a conocer en 1980, llamaba a abrir el concepto de seguridad para atender asuntos de desarrollo y bienestar social (Brandt Report, 1980).

Académicamente, el artículo parteaguas de Richard Ullman, Redefining Security, publicado en 1983, criticaba la visión estatal y céntrica que hasta entonces predominaba en los estudios de seguridad, sugiriendo a los estudiosos en la materia enfocar sus esfuerzos en el medio ambiente (Ullman, 1983). Para críticos como Michael Sheehan, lo más novedoso del argumento de Ullman fue que su artículo apareció publicado en la revista International Security, la cual es considerada como el veto exclusivo de los realistas (Sheehan, 2005: 45).

Sin embargo, no fue sino hasta el fin de la guerra fría que emergió una crítica mucho más radical y elaborada del concepto de seguridad internacional ortodoxo. Un académico, parafraseando la frase sobre las armas nucleares utilizada por John F. Kennedy, argumenta que el fin de la guerra fría cambió "todas las respuestas y todas las preguntas" (Kegley, 1993: 141). En efecto, el derrumbe soviético y la relativa ausencia de rivalidades hegemónicas pronto hicieron creer que el poder militar había dejado de tener la importancia que antes solía tener. Por breves momentos, las amenazas militares parecían menos predominantes, mientras que la fuerza militar se percibía como un instrumento menos útil para el Estado (lo que en inglés se conoce como statecraft). Por ejemplo, en 1989, John Mueller, un estudioso en la materia, afirmó que las guerras en los estados democráticos carecían de legitimidad, propósito y utilidad; por tanto, se esperaba que el conflicto armado entre estados se atrofiara en la medida en que los regímenes democráticos se establecieran mundialmente (Mueller, 1989).

1 Para una reseña histórica exhaustiva de la agenda de desarrollo económico y sus orígenes, incluyendo el debate sobre el Nuevo Orden Económico Internacional en el seno de la ONU, véanse Weiss et al. (2005) y Berthelot (2004). 
Estos hechos motivaron la emergencia de un movimiento de corte liberal que busca, en esencia, reexaminar la forma en que se piensan las relaciones internacionales, en general, y la seguridad internacional, en particular. Las propuestas para expandir el rango conceptual de la seguridad internacional están igualmente motivadas por la incapacidad de las explicaciones ortodoxas y realistas de anticipar la caída del bloque soviético y su impacto en la agenda de seguridad, así como la llegada del terrorismo transnacional y sus consecuencias. Como reclama David A. Baldwin, en un artículo ya clásico de World Politics, "los estudios de seguridad, como un campo académico, requieren una clarificación" (Baldwin, 1996: 117-141).

De tal forma, las propuestas para ampliar el estudio de la seguridad internacional se pueden dividir en dos tipos: las modestas y radicales. Para los seguidores de una reforma modesta del concepto, los estudios de seguridad deben dejar de lado el énfasis en la disuasión nuclear e incluir instrumentos políticos no militares. Dentro de esta clasificación, se puede incluir la obra de autores como Barry Buzan, Ole Waever y Jaap de Wilde, titulada Security: A New Framework for Analysis (Buzan, Waever y de Wilde, 1998). Los autores dividen el estudio de la seguridad sectorialmente, dando cabida a diferentes formas de seguridad, incluyendo la económica, social y política. Asimismo, sugieren incluir temas tales como la seguridad regional dentro del concepto que estos autores denominan como el complejo de seguridad; es decir, un grupo de estados cuyas preocupaciones y percepciones sobre seguridad no pueden ser analizadas o resueltas de manera independiente ni unilateralmente (Buzan, Waever y de Wilde 1998; Buzan, 1991).

Este tipo de enfoque es modesto en su intención y no sugiere una manera radical de estudiar la seguridad. Agrega elementos a los estudios clásicos sobre el tema, pero conserva una visión esencialmente estato-céntrica. Para Michael Sheehan y Martin Shaw, el intento de Buzán et al. por redefinir la agenda de seguridad internacional es insatisfactoria, ya que el enfoque permanece fiel a los supuestos básicos del realismo, cuyo énfasis está en el Estado, como actor primordial de las relaciones internacionales, y en la dualidad que caracteriza las relaciones estatales, la cooperación y el conflicto (Sheehan, 2005; Shaw, 1994).

En un segundo nivel están las propuestas más radicales, con agendas más amplias y con una crítica más explícita contra la visión ortodoxa de la seguridad. En esta línea de argumentación se encuentran autores tales como el propio David A. Baldwin, quien ha sugerido la abolición de los estudios de seguridad de una vez y para siempre, en virtud de que "la seguridad" engloba tantos temas que bien podrían incluir la totalidad de las ciencias sociales (Baldwin, 1997). Después de todo, el propio Karl von Clausewitz argumentaba que la guerra era parte de la política, pero con otros medios, con lo cual quería sugerir que incluso la guerra no debía ser estudiada de forma aislada de la política internacional (Clausewitz, 1976). Thomas Schelling, otro estudioso de la seguridad, invitaba a pensar la guerra en términos de procesos de negociación, en donde el conflicto y la cooperación eran inseparables (Schelling, 1960). Por tanto, las enseñanzas tanto de Clausewitz como de Schelling ya parecían sugerir la integración de los estudios de seguridad en ramas más amplias de la ciencia social. Entonces, ¿para qué llamarles estudios de seguridad, si en realidad son parte de otras disciplinas, como la economía y la sociología? Como sostiene 
el propio Baldwin, la integración de la seguridad internacional con otras disciplinas hace innecesario el tener una subdisciplina exclusivamente dedicada a asuntos militares, porque es tanta la interdependencia con otras ramas de estudio que se termina fragmentando el conocimiento de manera fútil (Baldwin, 1997).

Partiendo de esta crítica dura a los estudios ortodoxos, en la década de los noventa se desarrolló un concepto alterno, denominado seguridad humana. Se trata del intento liberal más reciente, elaborado y radical por ampliar el debate de la seguridad. Sobre la seguridad humana existe una muy abundante literatura, cuyo eje temático consiste en anteponer al individuo sobre el Estado, otorgando prioridad epistemológica al ser humano por encima de otras estructuras sociales y políticas. ${ }^{2}$ El concepto nace, en parte, por la insatisfacción que provocó la noción tradicional de seguridad internacional. Los proponentes de la seguridad humana ven en la versión clásica de la seguridad una visión unilateral, acotada, con énfasis en las armas y rivalidades militares entre estados, la cual además tiende a ignorar las amenazas ambientales, económicas y culturales (Bajpai, 2000). La seguridad así definida es inaceptable para los liberales, ya que en la mayoría de la veces los mismos estados son la fuente del problema más que la solución. ${ }^{3}$

Por tanto, liberados de los constreñimientos de la guerra fría, una plétora de instituciones internacionales, organizaciones no-gubernamentales y un grupo reducido de países considerados como potencias medias (Canadá, los países escandinavos e incluso Chile) ${ }^{4}$ propusieron el concepto de seguridad humana como una alternativa a la visión tradicional y ortodoxa hasta ese entonces predominante. ${ }^{5} \mathrm{~A}$ su alrededor se ha aglomerado una coalición sólida de agencias internacionales, organizaciones civiles y países que financian agendas políticas con el objetivo claro de desviar recursos de los temas de seguridad tradicional y así apoyar los temas no convencionales. Como sostiene Roland Paris, se trata de un concepto cuyo poder real radica en su capacidad de unificar a todo aquel que se considera contrario al tema militar y opuesto al realismo político. Se trata, pues, de un concepto que engloba a diferentes formas de liberalismo internacional (Paris, 2001: 87-102).

La idea principal detrás del concepto de la seguridad humana consiste en expandir la geografía y el sentido asociado al término "seguridad". Como describe Emma Rotschild en su reseña crítica e histórica del concepto, la expansión se presenta en cuatro formas. En su primera acepción, el concepto de seguridad es extendido a niveles inferiores; es decir, del nivel sistémico y estatal, baja hasta cubrir los niveles grupal e individual, de las naciones hasta los individuos. En su segundo significado, el término es expandido a un nivel superior, yendo desde los estados hasta el sistema internacional o supranacional,

2 Véanse, por ejemplo: Ogata y Cels (2003); King y Murria (2001); Thomas y Tow (2002), Suhkre (1999); Rojas y Gaucha (2001).

3 Véase Bellamy, Alex J. \& Matt McDonald (2002).

4 Otros estados que se unieron a la causa fueron Australia, Grecia, Irlanda, Jordania, Mali, los Países Bajos, Slovenia, Suiza y Tailandia.

5 El primer esfuerzo por oficializar el término surge en el seno de la Organización de las Naciones Unidas, particularmente el Programa de Naciones Unidas para el Desarrollo (PNUD) y su informe anual sobre Desarrollo Humano. Véase PNUD (1994). 
para cubrir la biosfera y hasta el medio ambiente. En su tercera distinción, la seguridad humana es expandida horizontalmente. Si el concepto ha de garantizar la subsistencia de varios entes y actores, entonces deberá extenderse de lo militar a lo político, económico, social y cultural. Finalmente, la responsabilidad política de proveer seguridad se amplía igualmente en todas direcciones para cubrir gobiernos locales y federales, organizaciones públicas y privadas, actores estatales, internacionales y no gubernamentales (Rotschild, 1995: 53-98).

El primer intento por poner en práctica este concepto consistió en el desarrollo de la doctrina de Naciones Unidas denominada "Responsabilidad de Proteger". Ésta está estructurada a partir del postulado de que la soberanía implica tanto derechos como responsabilidades. De tal forma, los estados están obligados a proteger a las poblaciones en situaciones de extrema vulnerabilidad, tales como guerras civiles, insurgencias, represiones, catástrofes naturales y colapsos estatales. Cuando un Estado es incapaz o no desea asumir su responsabilidad, ésta debe ser apropiada por la comunidad internacional, dando cabida a la intervención humanitaria. Como tal, la puesta en marcha de esta doctrina ofrece la posibilidad de que Naciones Unidas desempeñe un papel más amplio, en el cual los individuos tienen primacía sobre los estados miembros (ICISS, 2001). Para autores como Martha Finnemore, se trata de un cambio fundamental en la manera de operar y entender la seguridad internacional, cuyo origen está no en los asuntos militares, sino en las transformaciones normativas sobre soberanía y humanidad (Finnemore, 1996).

Consecuentemente, la seguridad humana puede comprender los siguientes elementos: seguridad económica y contra la pobreza, seguridad alimenticia, seguridad social y acceso a la salud, seguridad ambiental y protección contra la degradación, seguridad física y contra la delincuencia, seguridad comunal para garantizar la subsistencia de las minorías étnicas y culturales, y seguridad política para asegurar las libertades individuales. Esta visión intenta mejorar y ampliar el entendimiento sobre las múltiples vulnerabilidades que aquejan a la humanidad y tiende a expandirse en todas direcciones, cubriendo todas las áreas, temas y actores reales e imaginables (Kolodziej, 1992; Kolodziej, 2005).

\section{ANÁLISIS CONCEPTUAL Y EMPÍRICO: TRES RAZONES PARA NO AMPLIAR EL CONCEPTO DE LA SEGURIDAD INTERNACIONAL}

\section{Observación empírica versus desarrollo conceptual: la realidad internacional, ¿constante o cambiante?}

Se ha vuelto ya un lugar común el asociar los estudios de seguridad con el surgimiento de la guerra nuclear y la propia guerra fría. Esta asociación también ha inspirado el reclamo constante por modificar y ampliar el concepto de seguridad. Si la guerra frío terminó, ¿por qué no ha de cambiar el concepto que la determinó? No obstante, lo cierto es que la discusión académica y, si se quiere, política sobre el arte de la guerra ha estado presente desde mucho antes del conflicto bipolar. El estudio pionero de Quincy Wright, Study of War, publicado en 1942, es uno de los muchos ejemplos sobre la forma rigurosa y sistemática en que se estudiaban los temas de seguridad en la era preguerra fría (Wright, 1942). El 
libro, de hecho, fue la culminación de un proyecto de investigación mayúsculo que se inició en 1926 y que estaba influido por varios expertos, entre ellos William Fox, Arnold Wolfers, E.H. Carr y Bernard Brodie; este último, fundador de los estudios estratégicos en los Estados Unidos (Betts, 1997: 7-33).

Asimismo, el hecho de que el conflicto bipolar haya desaparecido no significa que los problemas provocados por la guerra hayan sido igualmente eliminados. La desaparición de la Unión Soviética no eliminó ni los medios militares (ahí están los arsenales nucleares, los ejércitos, persisten las industrias militares) ni los fines políticos de la guerra (Walt, 1991: 211-239). En términos de intensidad, se pueden distinguir los conflictos internacionales en tres tipos: de baja, mediana y alta intensidad. El espectro de conflictos, por tanto, cubriría desde la guerra de guerrillas en el nivel más bajo, hasta la guerra nuclear en el nivel más alto. De tal forma, los conflictos de baja intensidad cubrirían esencialmente las revoluciones armadas y las denominadas guerras de guerrillas. Existe una plétora de conflictos de este tipo, incluyendo Irak después de la ocupación estadounidense, la guerra interna en Colombia y el sinfín de conflictos internos en África subsahariana. Los conflictos de intensidad media incluirían las llamadas guerras convencionales, tales como el reciente conflicto entre Israel y el Líbano, los Balcanes, la primera guerra del Golfo y las guerras entre India y Pakistán. Finalmente, los conflictos de más alta intensidad cubrirían esencialmente las guerras entre las grandes potencias. ¿Cómo se han modificado estos diferentes tipos de conflictos en los últimos años?

Primero, es posible afirmar que la guerra ha sufrido pocos cambios en los conflictos de baja y mediana intensidad. El arte de la guerra es un viejo diablo que, como el buen vino tinto, entre más añejo mejor. A pesar de los muchos cambios tecnológicos y los avances en materia de equipamiento, lo cierto es que tanto la guerra de baja intensidad como la convencional reflejan pocos cambios en materia de estrategia militar. Es decir, la logística, operaciones y estrategia de la guerra parecen resistentes al paso del tiempo (Van Evera, 2001).

Históricamente, han sido pocas las revoluciones en materia bélica. La llamada visión revolucionaria de la guerra se desarrolló esencialmente como resultado del poder destructivo que representaban las armas nucleares, el cual, en última instancia, hacía de la guerra convencional algo imposible. No había forma de pelear una guerra convencional con armas nucleares. Ciertamente, este tipo de armamento revolucionó la forma en la que se entendían los escenarios bélicos y las hipótesis de guerra (Jervis, 1989).

Una segunda corriente geopolítica vio la expansión de movimientos guerrilleros como algo revolucionario en sí. Ciertamente, las guerrillas no eran novedosas para los ejércitos modernos, al menos no lo eran tácticamente. Las guerras de independencia habían sido esencialmente guerras de guerrillas. Lo novedoso, sin embargo, fue el surgimiento de movimientos guerrilleros después de la Segunda Guerra Mundial asociados con ideologías y pensamientos político-radicales. El reto en sí de la expansión de los movimientos revolucionarios no era táctico, sino político. Es decir, cuando los movimientos armados de guerrillas adquirieron un tinte político e ideológico que amenazaba la integridad política de un Estado. En especial, el pensamiento geopolítico europeo y estadounidense 
estaba preocupado por la expansión del Comunismo a través, precisamente, de guerras de guerrillas, como de hecho sucedió en China y hasta cierto punto en Grecia. ${ }^{6}$

En América Latina estas tesis sobre la visión revolucionaria no adquieren primacía sino hasta el triunfo de la Revolución cubana en el 1959, donde la amenaza revolucionaria se vuelve evidente tanto para los políticos como para los estrategas militares sudamericanos. Aquí hay una especie de variación en la conceptualización de los estados como entes orgánicos porque el pensamiento geopolítico ortodoxo del siglo XIX consideraba que la mayor amenaza provenía del exterior, de la posible expansión de otros estados. Para la visión revolucionaria, sin embargo, la mayor amenaza provenía esencialmente del interior de esos estados y sus patrocinadores externos. Es decir, en el Estado había células malignas que podían incluso ser tan dañinas como las amenazas externas (Child, 1985).

En la actualidad, se pueden identificar muy pocos cambios introducidos desde el fin de la guerra fría en materia de guerra revolucionaria o de baja intensidad. Probablemente la transformación más evidente sea en los términos, pero no en la forma. Si antes la guerra de guerrillas comunista era la mayor amenaza, ahora lo es la guerra de guerrilla terrorista. Nuevamente, el cambio es meramente de forma y semántico, porque los medios y las estrategias son esencialmente las mismas. Las fuentes de amenaza siguen siendo externas, provenientes de otros estados, e internas, provocadas por la subversión de grupos que no aceptan la autoridad política. ¡No hay, pues, nada nuevo bajo el sol!

Quizá ningún otro tema domina más los desplegados de los periódicos extranjeros y estadounidenses que la guerra en Irak. Se trata del clásico ejemplo de una guerra asimétrica y de baja intensidad, adonde el país con mayor capacidad militar se enfrenta contra diversos grupos guerrilleros que le llevan ventaja estratégica y política. Los problemas que hoy sacuden a los Estados Unidos no son muy distintos a los retos que alguna vez enfrentaran Francia y el Reino Unido en las ex colonias europeas en el Medio Oriente y Asia. Cualquiera que haya visto la película La Batalla de Argelia, en la cual se narran las confrontaciones entre Francia y la Argelia ocupada, podrá evocar escenas que parecen un recuento similar a lo que actualmente está sucediendo en el Medio Oriente. ${ }^{7}$ Los guerrilleros utilizan tácticas de miedo contra una población con el fin de modificar percepciones sobre la capacidad de control y gobernabilidad de las autoridades ocupantes. Los ocupantes, por su parte, responden con el medio que les ofrece supremacía -el militar- sabiendo que la solución es de carácter político. Por tal motivo, no es casual que autores como Jack Snyder sostengan que EUA es una potencia imperial, la cual, al igual que sus aliados en la vieja Europa, enfrenta esencialmente los problemas de cualquier imperio entrometido en una guerra civil: el ensanchamiento de sus compromisos extraterritoriales y la falta de legitimidad en aquellos territorios que ocupa (Snyder, 2003). En este sentido, no parece haber ninguna lección nueva sobre seguridad que la historia no haya proporcionado con anterioridad, sino políticos y líderes militares incompetentes que han preferido obviar las lecciones históricas.

6 Existe una vasta y abundante literatura sobre la guerra de guerrillas, pero puede consultarse la siguiente literatura: Osgood (1957), Blaufarb (1977) y Payne (1989).

$7 \quad$ La Battaglia di Algieri es una película dirigida por Pontevorco, Gillo, Roma, Italia, 1967, DVD, 125 minutos. 
Otro ejemplo concreto de guerras de baja intensidad son los conflictos que las Naciones Unidas han intentado solucionar a través de las misiones de paz. Es ya recurrente que la ONU se encuentre enfrascada en operaciones que jamás llegan a imponer una paz efectiva y duradera. El expediente en materia de derechos humanos puede ser igualmente cuestionable. Paradójicamente, las operaciones de paz tienden cada vez más a recurrir al uso de la fuerza militar, imponiendo, en la mayoría de los casos recientes, una paz precaria, como ha sucedido recientemente en el Congo, Sierra Leona y Haití (Lacey, 2005).

A pesar del desarrollo de la doctrina "Responsabilidad de Proteger", las dificultades que enfrenta la ONU para imponer la paz en contextos de guerra civil y étnica no son nuevas. Por principio, la forma en que se aplica la doctrina sigue siendo discrecional y usualmente está guiada por los intereses de las cinco potencias nucleares con derecho a veto en el Consejo de Seguridad. Por ejemplo, es ya sabido que el régimen político en Sudán ha cometido violaciones de derechos humanos y se le acusa, incluso, de cometer genocidio. No obstante, como es ya costumbre, el apoyo de uno de los miembros permanentes, China, al gobierno de Sudán ha impedido que el propio Consejo imponga sanciones más amplias al país africano, cuestionando la puesta en marcha de la doctrina "Responsabilidad de Proteger". 8

Por otro lado, la ONU enfrenta problemas ya harto conocidos en materia de intervención. Ya en la década de los sesenta la Organización intentó pacificar el Congo, justo después de su independencia de Bélgica. Sin embargo, la misión fracasó y, de hecho, en el intento por estabilizar el país, el Secretario General, Dag Hammarskjöld, falleció en un accidente aéreo en el Congo. La diferencia entre lo sucedido en los sesenta y lo que ocurre hoy es meramente de grado. La ONU hoy intenta resolver más de diez conflictos del estilo del Congo, incluyendo el propio Congo. ${ }^{9}$ En la mayoría de los casos, los cascos azules se enfrentan a movimientos insurgentes y guerrilleros (guerras de guerrillas o de insurgencia) que utilizan armamentos muy similares e incluso más arcaicos de los que se utilizaban hace dos décadas, como de hecho sucede en Haití. Como es ya sabido, el dilema que enfrenta la ONU consiste en involucrarse en conflictos donde las partes beligerantes perciben aún beneficios concretos por continuar la batalla y costos por dejar las armas.

De ahí que las misiones de paz sean muchas veces ceses al fuego temporales o treguas militares que perduran por décadas, como de hecho sucede en Chipre, Cachemira y Bosnia. Conflictos internos que se creían terminados resurgen a pesar de los acuerdos de paz, como de hecho sucede hoy en Haití, Timor del Este, Congo y, por supuesto, Líbano. ${ }^{10}$

$8 \quad$ Véase The Economist (2007) y (2004).

9 Durante el período de 1960-1990, el promedio de misiones de paz de la ONU era de no más de cinco operaciones por cada década. En contraste, para agosto del 2005, las Naciones Unidas estaban involucradas en 16 misiones de paz. Asimismo, el personal involucrado en esas operaciones de paz ha crecido sustancialmente. En 1947, el número de soldados desplegados en misiones de paz era de 30 observadores militares. Para 1978, el Consejo había ya autorizado el despliegue de 16.700 soldados de paz. En contraste, en 1993 (el año tope en materia de operaciones de paz), el número de militares involucrados en operaciones de paz se había incrementado a los 78.500, cuatro veces el tope máximo alcanzado durante el período de la guerra fría. En el 2006, el número se mantiene en 73.008 cascos azules. Véase Global Policy Forum (2006).

10 Para una visión crítica de las operaciones de paz de la ONU, véase Greig y Dile (2005). Una visión más liberal y optimista de las operaciones puede verse en Fortna (2004). 
Los viejos dilemas que encara la ONU en la actualidad hacen que el debate sobre la seguridad internacional parezca eso, vino viejo en botellas nuevas. ¿Qué hay de nuevo en la forma de operar de Naciones Unidas? Es probable que la doctrina "Responsabilidad de proteger" visualice una forma de operar distinta, pero, en la práctica diaria, los hábitos de la Organización parecen resistentes al cambio.

En segundo lugar, en materia de guerra convencional tampoco parecen ser evidentes las supuestas novedades. Si bien el número de conflictos entre estados se ha reducido sustancialmente, los gastos en presupuestos militares han aumentado a niveles superiores a los de la guerra fría. Los reportes anuales del Stockholm International Peace Research Institute revelan que los gastos son más altos y los conflictos más evidentes en regiones tales como el Medio Oriente y el Sur y el Este de Asia. ${ }^{11}$ Se trata de zonas geopolíticas que se siguen nutriendo del pensamiento militar convencional. Israel es el clásico ejemplo de un país que se prepara y se entrena para enfrentar una guerra en varios frentes con medios convencionales y no convencionales. Una situación similar sucede en Taiwán, donde los planes para una invasión china son meramente convencionales y el peligro mayor sigue siendo un ataque sorpresa de naturaleza convencional igualmente. El problema con este tipo de guerras no radica en el tamaño; es decir, cuántas armas, hombres y tanques se poseen, sino en el empleo eficiente de la fuerza (lo que en inglés se conoce como force employment). Los medios siguen siendo los mismos, quizá más modernos, pero en esencia los conflictos militares convencionales siguen teniendo las mismas características y objetivos de antaño: modificar el equilibrio de poder regional, impedir o revertir un dilema de seguridad; disuadir, constreñir y castigar al enemigo; evitar la dependencia política; obtener libertad e independencia política; erosionar a gobiernos débiles, y modificar los términos de la negociación. Como afirma Stephen Biddle en su más reciente obra, Military Power, los factores que llevan a la victoria en la guerra convencional son, básicamente, los mismos que en 1918: disimulo, encubrimiento, dispersión, supresión, maniobra y combinación de armas (Biddle, 2004). Aquí, nuevamente, se trata de vino viejo en botellas nuevas.

Paradójicamente, la vox populi, la cual incluye políticos y periodistas, insiste en visualizar al mundo como radicalmente diferente del que los antepasados cercanos vivieron. Ningún fenómeno parece atraer hoy más atención que el denominado terrorismo transnacional. La imagen del colapso de las torres gemelas en Nueva York, el 11 de septiembre del 2001, seguramente sigue acechando las mentes y memorias de millones de personas (y con razón). Los actos terroristas han marcado un parteaguas y sus efectos no parecen tener un precedente histórico.

La emergencia del terrorismo también ha generado una inquietud sobre la utilidad del viejo paradigma de la seguridad internacional, el cual es percibido como inservible para enfrentar las nuevas amenazas representadas por las propias organizaciones terroristas. Después de todo, el terrorismo es un tipo de violencia ejercida por actores privados, constituidos en organizaciones no estatales y descentralizadas, cuya misión cambia a través del tiempo; es

11 De acuerdo a las estadísticas reportadas por el SIPRI, el gasto militar mundial se ha elevado consecutivamente desde el 2002. El gasto militar en el 2005 representó un incremento real de 3,4 con relación al 2004 y 34\% en relación al período de 1996-2005. Las regiones que más gasto militar tienen son América del Norte, el Medio Oriente, Asia y Europa Central. Véase SIPRI (2006). 
decir, son funcionalmente opuestos al tipo de actores y organizaciones tradicionalmente analizados por los realistas y el pensamiento convencional sobre seguridad. Asimismo, los grupos terroristas poseen movilidad, están muchas veces asociados a religiones y movimientos fanáticos y utilizan medios convencionales y no convencionales (como podría ser internet). ${ }^{12}$ Por tanto, parece que el viejo paradigma basado en el modelo estatocéntrico es débil para entender las causas del terrorismo e incapaz para prescribir políticas que lo disuadan o eliminen.

Ciertamente, hay una verdad en estos argumentos, pero igualmente hay una realidad que no puede obviarse: los actos terroristas revelan el papel fundamental que los estados desempeñan en el sistema internacional. Como sostiene Robert Jervis, muchos de los movimientos hoy clasificados como terroristas (Al Qaeda, Hizbollah o Hamas) subsisten porque hay estados y gobiernos que los financian y mantienen. Sus prosélitos son reclutados y luego entrenados en territorios reclamados por algún Estado (Afganistán o Pakistán). Sus objetivos y sus misiones, usualmente, están dirigidos contra un estado. E, infelizmente, muchos de esos movimientos se originan en respuesta no a la pobreza o la globalización, sino en reacción a las políticas exteriores de los propios estados, especialmente los más poderosos. ${ }^{13}$ Curiosamente, la respuesta frente a la amenaza del terrorismo ha sido, precisamente, reforzar los medios estatales tradicionales: ampliación de los servicios estatales de inteligencia e incremento de presencia y gasto militares (Jervis, 2002: 37-54). ${ }^{14}$ ¿Dónde está, pues, la novedad? ¿Qué hay de nuevo en el terrorismo transnacional que no se haya presenciado con anterioridad? Más allá del temor generalizado y expandido por los medios y el grado de la amenaza, ¿qué hay de nuevo en materia de estrategia, operaciones, logística y análisis de seguridad?

Ahora bien, el lector seguramente se preguntará por qué parece existir tan poca novedad en el ámbito de la seguridad internacional. Parte del problema quizá esté asociada al razonamiento de los tomadores de decisión. Los individuos no son tomadores de decisiones perfectamente racionales. Éstos deben decidirse bajo presión externa, con poco tiempo disponible para meditar bien las alternativas militares. De tal forma, los líderes políticos se hacen valer de diversas técnicas para evaluar y procesar la información. La mayor parte del tiempo utilizan su percepción, algunas veces basados en experiencias anteriores. Así una experiencia actual, como la guerra en Irak, les evoca una anterior, la guerra en Vietnam, y de ahí establecen una relación. Frente a diversas crisis internacionales, los líderes comprenden la realidad en la medida en que se relaciona con otros momentos del pasado. En otras palabras, aceptan la información que es compatible con la información que ya ha sido previamente canalizada y admitida, en tanto que ignoran la información que es inconsistente con alguna experiencia previa. Por tanto, los tomadores de decisión suelen recaer en procesos cognitivos que, en última instancia, informan su proceso de toma de decisiones. ${ }^{15}$ 
Por ejemplo, el grupo de tomadores de decisión detrás de la administración del Presidente George W. Bush son, en su mayoría, viejos veteranos de los gobiernos de Gerald Ford y Richard Nixon. Por tanto, no es de extrañar que en muchos de los casos se intente resolver problemas de la misma forma en que Ford y Nixon tomaban decisiones durante la guerra fría. Frente a lo incierto, los líderes parecen preferir caminos ya conocidos, para lo cual la estrategia militar ortodoxa parece ser la mejor receta. No obstante, como explica el propio padre de la psicología-política internacional, Robert Jervis, los factores estructurales, estratégicos y de seguridad no pueden ser obviados. "Bajo la doctrina Bush, los Estados Unidos han dejado de ser una potencia statu quo. Sus motivos pueden no ser egoístas, pero la combinación de poder, miedo y oportunidad percibida llevan al país a intentar reconfigurar la política mundial y la gran mayoría de los miembros de la sociedad que la conforman" (Jervis, 2003: 375).

Tercero, resulta extraño que los verdaderos cambios, quizá los más fundamentales, suelen ser inadvertidos por la gran mayoría. El área adonde parece existir un verdadero enigma es el de los conflictos de alta intensidad. Las guerras entre las grandes potencias han dejado de ser fenómenos recurrentes de la seguridad internacional. Este es un hecho paradójico porque la atención de la comunidad académica y la de los tomadores de decisión parecen estar volcadas exclusivamente sobre los conflictos internos y los movimientos guerrilleros, obviando el hecho de que el cambio más radical está en materia de conflictos de alta intensidad. Por qué las potencias mundiales han perdido interés en confrontarse unas con otras es un verdadero enigma que hasta ahora no se ha explicado cabalmente y que los nuevos conceptos de seguridad humana tampoco parecen reconocer. ${ }^{16}$

No obstante, la aparente paz perpetua entre potencias militares no justifica, en sí misma, una reconceptualización del término seguridad internacional. Por principio, la falta de conflicto armado no significa que el mundo esté más seguro de amenazas nucleares y militares. Como sostiene el propio David A. Baldwin, en el debate conceptual no hay que confundir la observación empírica con el proceso de especificación conceptual (Baldwin, 1997: 5-26). Que una parte de la realidad internacional haya cambiado no justifica mudar o erradicar conceptos. El análisis conceptual conlleva clarificar significados, operacionalizarlos en su más amplio sentido y referirlos o vincularlos a teorías vigentes. Asimismo, aun si sólo se considera la realidad empírica, resulta evidente que las grandes potencias no han renunciado al uso de la fuerza y están dispuestas a amenazar e incluso atacar enemigos más débiles militarmente.

Es verdad que la versión de seguridad internacional ortodoxa, basada en temas estratégicos y militares, parece acotada, exclusiva y restrictiva. Pero un concepto no se invalida por ser acotado, sino porque carece de especificidad y operacionalidad. La tendencia de incluir otras formas de amenazas, medios y formas al concepto de seguridad internacional puede conducir a la confusión conceptual y por tanto a la prescripción de políticas incorrectas cuando no se especifican ni sus objetivos ni sus medios. Si bien es cierto que las grandes potencias militares no se enfrentan más entre sí, éstas siguen valorando su seguridad

16 Ha habido algunos intentos teóricos para explicar este aparente enigma, aunque ninguno ha sido lo suficientemente convincente, véase: Jervis (2002b), Pape (2005), Lieber y Gerard (2005). 
estatal, privilegian los medios militares y perciben aun amenazas provenientes de otros Estados. El concepto tradicional, por tanto, sigue teniendo vigencia y aplicabilidad a pesar del cambio internacional.

Consecuentemente, la seguridad internacional en la Postguerra Fría y postseptiembre 11 ofrece pocas novedades y muchas continuidades. Los principios y definiciones de la seguridad internacional ortodoxa y tradicional, bajo los términos del realismo, parecen erigirse como una vieja institución bien establecida a la cual se retorna irremediablemente más tarde que temprano, sobre todo cuando hay un cañonazo. De tal forma, los estudios disciplinarios sobre estrategia militar, dentro de la seguridad internacional, son difíciles de evadir e imposibles de ignorar.

\section{Militarismo y los peligros del olvido}

Las propuestas liberales dispuestas a redefinir las fronteras de lo que es y no es seguridad internacional tienden a reducir la relevancia de los asuntos militares y estratégicos. Aun si se acepta la idea de que los estudios militares han dejado de ser importantes, lo cual es cuestionable en sí, surge la duda sobre quién debe analizar los temas de estrategia militar. El problema con las propuestas liberales, como la seguridad humana, es que los temas militares quedan rezagados a tal punto que se vuelven un auténtico chícharo (o guisante) en una sopa amorfa de verduras. Entre tanta verdura no se advierte si el chícharo está podrido o crudo, simplemente se deja y abandona hasta que causa estragos estomacales. De hecho, este debate ya había sido resuelto hace décadas, cuando en 1949 el propio Bernard Brodie escribió el ya clásico Strategy as Science (Brodie, 1949). Brodie tenía en mente una ciencia instrumental que resolviera los problemas prácticos del arte de la guerra. Hasta entonces muchos académicos reconocían la importancia de la guerra, pero parecían estar interesados exclusivamente en el antes y el después, no en la guerra en sí. Para Brodie, los estudios estratégicos debían incluir las tres fases: el antes, durante y después de un conflicto bélico. Brodie quería evitar precisamente la calidad superficial que hasta ese entonces prevalecía en los estudios sobre seguridad internacional. Como argumenta Richard Betts, Brodie hablaba como el George Clemenceau de la academia: para este pensador político, como para Clemenceau, los asuntos de estrategia militar eran demasiado importantes como para ser dejados en manos de generales (Betts, 1997).

En efecto, las propuestas liberales y radicales para reformar y cambiar los estudios de seguridad son la receta idónea para dejar los asuntos de la guerra y la paz en manos de unos cuantos uniformados. Los problemas que tanto temía Brodie en 1949 bien pueden replicarse en la actualidad. Es decir, reproducir análisis superficiales sobre la guerra y la paz que incluyen todo y nada al mismo tiempo.

De hecho, probablemente en ninguna otra región del mundo se aprecian mejor los efectos perversos de la expansión del concepto de seguridad internacional que en América Latina. En esta parte del mundo, los presupuestos militares per cápita son relativamente bajos, el debate político-social ha estado influido por los dilemas del bienestar social, la consolidación democrática, el Estado de derecho, la justicia social, la corrupción 
institucional y la seguridad pública. ${ }^{17}$ Las encuestas desde Brasil hasta México, pasando por El Salvador e incluso Chile, sostienen, sistemáticamente, que el crimen organizado y la seguridad pública se han vuelto el reto más importante. ${ }^{18}$ Los partidos políticos y las organizaciones sociales, justificadamente, se han abocado a enfrentar y estudiar estos fenómenos sin encontrar aún una solución viable.

Sin embargo, es precisamente en este renglón donde los estudios estratégicos y de seguridad ortodoxos se hacen más necesarios. Tanto en países grandes (Brasil y México) como en los chicos (El Salvador) y medianos (Colombia y hasta Chile), las autoridades públicas utilizan a las fuerzas armadas para mantener el orden público, ahí cuando fallan las otras instituciones públicas. Ya sea para enfrentar al narcotráfico, para disuadir a grupos guerrilleros, para mantener el orden público o para salvar vidas frente a catástrofes naturales, las fuerzas armadas de la región son constantemente llamadas a desempeñar funciones para las que tradicionalmente no fueron ni creadas ni desarrolladas. Frente a la plétora de problemas sociales (la gran sopa de verduras) en América Latina, que incluye seguridad pública y vial, seguridad ciudadana y seguridad comunitaria, la seguridad estratégica se ha vuelto un diminuto chícharo al cual nadie pone atención más que los propios uniformados. Sólo los militares tienen centros de estudios estratégicos y sólo ellos entrenan y doctrinan sin que los ciudadanos y académicos impongan límites y fronteras disciplinarias. Desde el retorno a la democracia en la región hace ya más de dos décadas, la reforma militar y la modernización de las fuerzas armadas ha importado sólo a unos cuantos porque el resto parece estar convencido de que la agenda de seguridad es tan expansiva y amplia que los militares no merecen prioridad alguna, al menos no frente al resto de los problemas sociales. América Latina entonces tiene la peor de todas las combinaciones: indiferencia ciudadana frente a la cuestión militar, fuerzas armadas mal entrenadas e ineficientes, gastos en defensa superfluos e irracionales, inseguridad estratégica y crisis diplomáticas constantes, por no mencionar la carencia de instituciones internacionales sólidas en materia de seguridad regional e internacional. Cabe preguntarse si esta crisis de seguridad no se agudizaría aún más con una agenda expansiva y radical, como la que proponen los seguidores de la seguridad humana.

Paradójicamente, la expansión conceptual puede llevar, igualmente, al efecto contrario del descrito arriba. Es decir, en el intento por incluir más temas dentro de la seguridad internacional, existe el peligro de "seguritizar" la agenda y eventualmente militarizarla. Para autores como Daniel Deudney, Richard Matthews y Jef Huysmans, el concepto de seguridad ha estado tradicionalmente dominado y cargado por los temas militares y estratégicos; ello implica que se puede llegar a imponer este marco de referencia sobre los temas que no son de corte militar (Deudney y Matthews, 1999 y Huysmans, 1998). Por ejemplo, un tema tradicionalmente social, como la migración internacional, paulatinamente

17 El porcentaje del PIB destinado para el gasto militar es relativamente bajo para América Latina (1,5 en promedio), con excepción de Colombia y Chile, cuyo gasto militar en relación al PIB es de 4,2 y 3,9, respectivamente. En términos reales, Brasil es el país que más gasto militar tiene en la región, aunque en proporción al PIB éste sólo representa el 1,6\%. Véase SIPRI (2006).

18 De acuerdo a las recientes estadísticas reportadas por Latinobarómetro, el $30 \%$ de los latinoamericanos considera que el desempleo es el problema más grave, seguido por un $14 \%$ de la delincuencia/seguridad pública y $9 \%$ la inflación y demás problemas políticos. Véase Latinobarómetro (2005). 
ha sido incorporado a la agenda de seguridad internacional, sin que ese enfoque lleve a entender o resolver mejor el fenómeno. ${ }^{19}$ En Estados Unidos, el once de septiembre ha provocado un debate amplio sobre cómo y cuándo se debe permitir la entrada de extranjeros para no arriesgar la seguridad interna. Sin embargo, la orientación que ha dominado hasta ahora la agenda migratoria estadounidense ha sido, precisamente, la militar. Literalmente, la reforma migratoria ha sido "seguritizada" por el Congreso y los movimientos anti-migratorios, quienes han sugerido incluso la militarización de la frontera sur con México, para impedir el cruce de ilegales hacia los Estados Unidos. El Presidente George W. Bush ha autorizado ya el despliegue de efectivos de la Guardia Nacional en la frontera y se construye un muro en el borde con México que emula un tipo de defensa militar para impedir el paso de un supuesto enemigo. ${ }^{20}$ Con certeza se puede afirmar que ninguna de esas dos medidas impedirá o restringirá el flujo migratorio, porque la esencia del problema no es militar y tampoco debe ser tratado como un asunto de seguridad, sino como uno de desarrollo económico y laboral.

De tal forma, el estatus intelectual e institucional de los estudios de seguridad está confundido por la persistente ausencia de consenso sobre cuánta atención deben recibir los aspectos militares y dónde se deben demarcar las líneas fronterizas. Un comentario semántico sobre el término "seguridad" es bienvenido. Los argumentos relacionados con la inclusión de temas económicos, ambientales y migratorios en la agenda de seguridad son justos y necesarios. Sin embargo, estos debates semánticos no permiten organizar el campo de las relaciones internacionales, porque en sí mismas no delimitan una subdisciplina. Ésta debe ser amplia como para incluir un rango suficiente de problemas, pero lo suficientemente acotada como para tener coherencia y distinguirse de las demás subdisciplinas. Las recientes propuestas por expandir la noción de seguridad, como lo es la seguridad humana, hacen indistinguible cualquier tipo de disciplina o subdisciplina. No resuelven el problema de las fronteras entre áreas de estudio y sí plantean problemas metodológicos, de puesta en marcha y de políticas públicas. Si acaso, lo que se requiere es crear más subdisciplinas, como medio ambiente, derechos humanos y migración, en lugar de eliminar los estudios de seguridad o acotar los de estrategia militar.

Muchas veces, la aversión por los estudios estratégicos y militares tiene su origen en las sospechas morales y los juicios de valor. Después de todo, la guerra es un acto que para muchos es inmoral y no debería existir. Para otros, la guerra está asociada con el pensamiento realista y su énfasis en el poder militar, lo cual repugna a quienes se sienten afines con el liberalismo y el pacifismo. No obstante, el objeto de análisis de los estudios de seguridad está igualmente concentrado en discernir las formas de evitar una guerra. De hecho, contrario a la creencia convencional, la mayor parte de los trabajos sobre estrategia militar son profundamente conservadores, como conservador es el pensamiento realista. Son pocos los estrategas, como pocos los realistas también, quienes prescriben la guerra como el medio idóneo o el primer medio a ser utilizado frente a una crisis internacional. Al contrario, la atención está enfocada en asuntos relacionados con la disuasión o la manera 
de contener la violencia armada para producir cambios (Art. 1980). Paradójicamente, son los liberales quienes están mucho más dispuestos a hacer uso de la fuerza militar para alcanzar otras metas políticas. No es casual que el derecho de intervención humanitaria sea defendido por los seguidores de los derechos humanos, como tampoco es coincidencia que los ataques preventivos sean abrazados por un grupo reducido de ideólogos en Washington que están convencidos sobre los efectos positivos de la democratización vía la fuerza. ${ }^{21}$

\section{El ensanchamiento conceptual y la prescripción errática: los problemas de la seguridad humana}

La seguridad humana, como concepto alterno al tradicional y ortodoxo, padece de dos problemas. Primero, la seguridad humana sufre de un viejo mal que aqueja a las ciencias sociales: el ensanchamiento conceptual. El analista chileno Francisco Rojas defendía la seguridad humana porque el contexto internacional demandaba "perspectivas holísticas. Es decir, la agregación de variables ubicadas en una perspectiva con ambición totalizadora" (Rojas, 2001: 18). Curiosamente, hace ya casi cuatro décadas Giovanni Sartori informaba sobre los riesgos inherentes en el desarrollo de conceptos holísticos, universales, expansivos e inclusivos. En efecto, la pretensión por cubrir toda la realidad en un solo concepto es irreal; toda teoría y por ende los conceptos que la rodean siempre serán sólo una fracción de la realidad. Ésta siempre cubre más hechos y es más amplia y compleja que cualquier concepto existente. Al ejercicio de ampliación y expansión de la investigación dispuesta a cubrir más mundo y realidad, Sartori lo denominó como ensanchamiento conceptual. Sostenía el filósofo-político italiano que los beneficios por ampliar conceptos, significados y aplicaciones son contrarrestados por las pérdidas en precisión y connotación. Argumentaba Sartori que: "Aparentemente, se cree que uno puede cubrir más -en términos de viaje- pero sólo se dice menos y con menor precisión... lo conduce a uno hacia la conceptualización indefinida y lo no delimitado. No se ha ganado nada si nuestros universales se han vuelto en categorías indiferentes que conducen a las pseudoequivalencias"' (Sartori, 1970).

La seguridad humana es el más reciente ejemplo vívido de ensanchamiento conceptual en las relaciones internacionales. En su esfuerzo por cubrir todo el espectro de áreas que ponen en riesgo al ser humano, se sugiere un concepto supuestamente holístico, pero a fin de cuentas indeterminado. Efectivamente, la seguridad humana envuelve desde el abuso contra mujeres hasta el genocidio, e incluye igualmente seguridad física y de otras formas, como la económica y cultural. Tan expansivo se ha vuelto el concepto de seguridad que resulta imposible determinar cuáles son las variables y factores excluidos. Todo indicador que ponga en riesgo y amenace la vida humana -incluyendo un accidente automotriz o el mero hecho de abordar un avión, por no hablar de la seguridad laboral, sin la cual no se tiene ingreso para alimentar-puede ser considerado como parte de la seguridad humana y por tanto internacional. $\mathrm{Si}$, en efecto, todo se puede incluir en un único concepto, entonces qué lo define sino la indeterminación del objeto y sujeto de estudio. 
Aún más, todo ejercicio conceptual conlleva la tarea de delimitar las categorías o clases que habrán de ser consideradas dentro del concepto en cuestión. Después de todo, el proceso de conceptualización es anterior al de medición y operacionalización de una teoría y antes de medir se debe especificar lo que está siendo medido. Para ello, las clases y categorías de un concepto deben ser exclusivas. Es decir, se requiere delimitar los fenómenos a estudiar y por ende indicar aquellos que se habrán de excluir, especificando fronteras y límites. Por esa razón, todo concepto es una especie de contenedor de datos, posee información sobre ciertos fenómenos distintivos y específicos, aunque excluye información sobre otros eventos y hechos. No obstante, la pretensión de la seguridad humana es precisamente lo contrario: lejos de delimitar, excluir y definir, el concepto incluye, indetermina, sin precisar. Si en el concepto de seguridad humana cabe todo lo que amenaza a la raza humana, entonces no se estudia nada en concreto.

Segundo, existe el problema vinculado a la puesta en práctica del concepto. La indeterminación conceptual en la cual se encuentra enfrascada la seguridad humana problematiza su aplicación al hacer difícil la identificación de causas y efectos concretos. Como argumenta Roland Paris, es impráctico y metodológicamente incorrecto hablar sobre factores socioeconómicos como los causantes o determinantes de la seguridad humana, en virtud de que esos son precisamente los elementos que forman parte de la definición misma del concepto (Paris, 204: 371). Ciertamente, la seguridad humana propone una tautología en donde las variables dependientes y los indicadores de un aumento o ascenso de la seguridad están determinados por las mismas variables causales que además definen el concepto (la causa y el efecto terminan siendo lo mismo), llevando, por tanto, a sugerir un argumento circular.

$$
\begin{aligned}
& \text { Causas de la inseguridad humana } \longrightarrow \text { Hambre, pobreza, degradación. } \\
& \text { Efectos de la inseguridad humana } \longrightarrow \text { Más hambre, pobreza y degradación. } \\
& \text { Solución de la inseguridad humana } \longrightarrow \text { Nutrición, desarrollo y conservación. } \\
& \text { Definición de seguridad humana } \longrightarrow \text { Inseguridad alimenticia, social y } \\
& \text { ambiental. }
\end{aligned}
$$

Si la solución al problema de la inseguridad es la mismo que su causa, entonces no sólo es difícil analizar el concepto, desagregarlo y entenderlo, sino además se hace imposible proponer soluciones coherentes y mutuamente compatibles. Por lo tanto, la definición de la seguridad humana es amplia y vaga como para ser significativa para los tomadores de decisión, ya que envuelve un rango amplio y diferente de amenazas. Al mismo tiempo, el concepto presenta graves problemas metodológicos para los académicos, ya que prescribe soluciones diversas y hasta incompatibles.

Adicionalmente, el concepto delega e ignora los problemas de seguridad estratégica, con lo cual se plantean otros problemas que bien podrían afectar la propia seguridad humana. 
Si un Estado es incapaz de garantizar la subsistencia política en el sistema internacional, cabe preguntarse si sería igualmente inerme para asegurar la seguridad individual de sus ciudadanos. Sin el Estado, el ciudadano es inexistente y sin su presencia es imposible velar por las garantías políticas y sociales, en virtud de que no habría un contrato social, por principio. La seguridad estratégica no es sólo vital para el Estado, también lo es para los individuos y la sociedad civil. La indiferencia con la cual la seguridad humana trata a los temas estratégicos y militares crea un problema cívico-militar, porque una ciudadanía indiferente frente a los temas tradicionales de seguridad y defensa es la receta ideal para que el Estado y sus instituciones sean igualmente indiferentes frente a los intereses sociales e individuales. Un concepto que sugiere olvidar los temas militares y de defensa, implícitamente propone dejar esos temas en manos de los generales y sin intervención civil. Paradójicamente, quizá sin darse cuenta de ello, la seguridad humana sugiere militarizar los problemas no militares y eso, para quienes aún creen en los estudios estratégicos y de seguridad tradicional, sería una grave amenaza a la seguridad nacional e internacional.

\section{CONCLUSIONES}

Las expectativas sobre una supuesta revolución en asuntos militares y un cambio normativo en la agenda de seguridad internacional reflejan, por un lado, una lectura inadecuada de la historia militar y, por otro, ofrecen igualmente una prescripción peligrosa para la actual política mundial. El peligro radica en profesar una fe ciega en la revolución científica y liberal que puede fácilmente llevar a sobreestimar el papel de la nueva tecnología y los nuevos retos, en tanto que se subestiman los factores que simple y llanamente no han sufrido cambios radicales desde el final de la guerra fría, como podrían ser la guerra convencional y la guerra de guerrillas.

Asimismo, no sólo los intentos por expandir los términos del debate conceptual son prematuros, sino que además están saturados de una carga normativa que pretende redefinir las agendas de política internacional en lugar de nutrir la discusión teórico-conceptual. Así, por ejemplo, se busca incluir dentro de la agenda de seguridad internacional temas de derechos humanos, económicos y ambientales, así como temas de equidad y justicia social. En el fondo, estas propuestas poseen una combinación de argumentos normativos y están cargados de adjetivos, los cuales en sí mismos sólo intentan jerarquizar juicios de valor sobre los derechos de los individuos, sin mostrar empírica y teóricamente la naturaleza y magnitud con la que deben ser medidos e incorporados esos temas al debate conceptual de la seguridad internacional. En términos de políticas públicas, las propuestas por ampliar el debate conceptual suenan razonables e incluso deseables. En términos metodológicos y de puesta en marcha, dichas propuestas son débiles, difíciles y hasta imposibles de poner en práctica. En otras palabras, la finalidad de quienes intentan expandir el concepto parece política y no heurística.

Los temas ortodoxos y tradicionales de la seguridad internacional son relevantes no sólo para los militares, sino para el Estado y la sociedad civil en general. Abandonar su estudio y anteponerlo a temas vinculados al desarrollo económico es una propuesta que unifica a 
pacifistas y liberales, pero plantea serios problemas y riesgos. En la vieja agenda se incluían los temas tradicionales como estrategia, guerra, operaciones y logística. Estos asuntos eran importantes no sólo por ser temas bélicos, sino porque se creía que en la medida que se entendiera la guerra, ésta podía ser evitada. En esta agenda también se incorporaban temas sociales, como eran las relaciones cívico-militares, el control civil sobre las fuerzas armadas, justicia y derecho de guerra, negociación internacional y la vinculación entre diplomacia y el uso de la fuerza. Paradójicamente, hoy el interés por estos asuntos parece difuso, justo cuando su estudio se hace más necesario. Tal parece que el viejo adagio de Clemençeau nos persigue por doquier: la guerra es demasiado importante como para dejársela a los generales.

Por otro lado, los problemas que emanan por el hambre, la discriminación de raza y género, degradación ambiental, migración e incluso las pandemias -todos graves y merecedores de atención- deben ser vistos, propiamente, como problemas del desarrollo y no de la seguridad. Cuando el Estado puede resolverlos, entonces forman parte del estado de bienestar y cuando requieren cooperación internacional, forman parte de la cooperación internacional para el desarrollo. Por tanto, ¿por qué desarrollar neologismos cuando se tienen conceptos que describen perfectamente la problemática? ¿Por qué expandir la seguridad internacional, cuando se trata de temas vinculados al desarrollo internacional? ¿Por qué suplir e incluso tratar con negligencia la seguridad estratégica y militar?

Quizá lo que habría que explicar es por qué el modelo que otorgaba énfasis en la seguridad individual perdió vigencia frente al modelo estatocéntrico. En la medida en que se identifiquen las condiciones que impidieron el desarrollo pleno del modelo individual en el pasado se podrá inferir si los nuevos reclamos a favor de la seguridad humana tendrán un futuro prometedor o si terminarán como los viejos esfuerzos: en ideas jamás concretadas.

\section{REFERENCIAS}

Allison, Graham y Phillip Zelikow. 1999. Essence of Decision: Explaining the Cuban-Missile Crisis. New York: Longman.

Art, Robert. 1980. "To What Ends Military Power". International Security 4(1): 4-35.

Bajpai, Kanti. 2000. "Human Security: Concept and Measurement". Kroc Institute Occasional Paper. \#19: OP: 1, Notre Dame, Indiana: University of Notre Dame. [En línea] http: / / www.nd.edu/ krocinst/ ocpapers/op_19_1.PDF\#search='human\%20security [Consulta 22-10-2007]

Baldwin, David.1996. "Review Article: Security Studies and the End of the Cold War". World Politics 48(1): 117-141.

Baldwin, David. 1997. "The Concept of Security". Review of International Studies 23(1): 5-26.

Bellamy, Alex y Matt McDonald. 2002. "The Utility of Human Security: Which Humans? What Security? A reply to Thomas \& Tow". Security Dialogue 33(3): 373-377.

Berthelot, Yves. (ed). 2004. Unity and Diversity in Development Ideas: Perspectives from the UN Regional Commissions. Bloomington, Indiana: Indiana University Press.

Betts, Richard. 1997. "Should Strategic Studies Survive?". World Politics 50(1): 7-33.

Biddle, Stephen. 2004. Military Power: Explaining Victory and Defeat in Modern Battle. Princeton, Nueva Jersey: Princeton University Press.

Blaufarb, Douglas. 1977. The Counterinsurgency Era. New York: Free Press. 
Booth, Ken. 1991. "Security and Emancipation". Review of International Studies (17): 313-326.

Brandt Report. 1980. Report of the Independent Commission on International Development Issues, North-South Programme for Survival. London: Pan Books.

Brodie, Bernard. 1949. "Strategy as Science". World Politic 1(4): 467-488.

Buzan, Barry, Ole Waever y Jaap de Wilde. 1998. Security: A New Framework for Analysis. Boulder, Colorado: Lynne Rienner.

Buzan, Barry. 1991. People, States, and Fear: An Agenda for International Security Studies in the Post-Cold War Era. Boulder: Lynne-Rienner.

Child, Jack. 1985. Geopolitics and Conflict in South America: Quarrels Among Neighbors. Nueva York: Praeger.

Clausewitz, Karl Von. 1976. On War. Princeton: Princeton University Press.

Cronin, Audrey Kurth. 2002. "Behind the Curve: Globalization and International Terrorism". International Security 27(3): 30-58.

Deudney, Daniel y Richards Matthews. (eds). 1999. Contested Grounds: Security and Conflict in the New Environmental Politics. Albany: State University of New York Press.

Finnemore, Martha. 1996. "Constructing Norms of Humanitarian Intervention”. En The Culture of National Security, editado por P. Katzenstein. Nueva York: Columbia University Press, 153-184.

Fortna, Virgina. 2004. "Interstate Peacekeeping: Causal Mechanisms and Emprical Effects". World Politics 56(4): 481-589.

Gause III, Gregory. 2005. “Can Democracy Stop Terrorism?”. Foreign Affairs 84(5): 62-76.

Global Policy Forum. 2006. Size of UN Peacekeeping Forces: 1947-2006. [En línea] <www.globalpolicy.org/ security/peacekpg/data/pcekprs.htm.> [Consulta 22-10-2007].

Greig, J. Michael y Paul Dile. 2005. "The Peacekeeping-Peacemaking Dilemma". International Studies Quarterly 49(4): 621-645.

Huysmans, Jef. 1995. "Migrants as a Security Problem: Dangers of Securitizing Societal Issues". En Migration and European Integration: The Dynamics of Inclusion and Exclusion, editado por R. Miles y D. Thranhardt. Londres: Pinter, 53-72.

Huysmans, Jef. 1998. "Security! What Do You Mean? From Concept to Thick Signifier". European Journal of International Relations. 42(2): 226-255.

ICISS (International Commission on Intervention and State Sovereignty). 2001. The Responsibility to Protect. Ottawa, Canadá. International Development Research Center.

Jervis, Robert. 1976. Perception and Misperception in International Politics. Princeton, Nueva Jersey: Princeton University Press.

Jervis, Robert. 1989. The Meaning of the Nuclear Revolution: Statecraft and the Prospect of Armageddon. Ithaca: Cornell University Press.

Jervis, Robert. 2002. "Theories of War in an Era of Leading-Power Peace: Presidential Address". American Political Science Review 96(1): 1-14.

Jervis, Robert. 2002b. "An Interim Assessment of September 11: What Has Changed and What Has Not?". Political Science Quarterly 117(1): 37-54.

Jervis, Robert. 2003. "The Uses of American Power: Explaining the Bush Doctrine". Political Science Quarterly. 118(2): 365-388.

Kegley, Jr. Charles W. 1993. “The Neoidealist Moment in International Studies? Realist Myths and the New International Studies". International Studies Quarterly 37(2): 131-146.

King, Gary y Christopher J Murria. 2001. “Rethinking Human Security”. Political Science Quarterly 116(4): $585-61$.

Kolodziej, Edward. 1992. "Renaissance in Security Studies? Caveat Lector!” International Studies Quarterly 36(4): 421-438.

Kolodziej, Edward. 2005. Security and International Relations. Nueva York: Cambridge University Press. La Battaglia di Algieri, dirigida por Pontevorco, Gillo, Roma, Italia, 1967, DVD, 125 minutos.

Lacey, Marc. 2005. "U.N. Forces Using Tougher Tactics to Secure Peace”. New York Times. Mayo 23, 2005. Sección A, p. 1. 
Latinobarómetro, Informe 2005, Santiago, Chile [En línea] <www.latinobarometro.org/uploads/media/2005. pdf> [Consulta: 10-4-2007]

Lieber, Keir y Gerard Alexander. 2005. "Waiting for Balancing: Why the World Is Not Pushing Back". International Security 30(1): 125-139.

McDermott, Rose. 2004. Political Psychology in International Relations. Michigan: University of Michigan Press.

Mueller, John. 1989. Retreat from Doomsday. Nueva York: Harper-Collins.

Ogata, Sadako y Johan Cels. 2003. "Human Security-Protecting and Empowering the People". Global Governance 9(3): 273-282.

Osgood, Robert. 1957. Limited War, Chicago: University of Chicago Press.

Pape, Robert. 2003. "The Strategic Logic of Suicide Terrorism". American Political Science Review 97(3): $1-19$.

Pape, Robert. 2005. "Soft Balancing against the United States". International Security 30(1): 36-45.

Paris, Roland. 2001. "Human Security: Paradigm Shift or Hot Air?". International Security 26(2): 87-102.

Paris, Roland. 2004. "Still an Inscrutable Concept". Security Dialogue 35(3): 370-372.

Payne, Jr., Samuel. 1989. Revolutionary Guerrilla War. The Conduct of War: An Introduction to Modern Warfare. Oxford: Basil Blackwell.

Philips, Kate. 2007. "The Immigration Debate". New York Time, Mayo 18, Sección US politics. [En línea]. <http: //thecaucus.blogs.nytimes.com/2007/05/18/the-immigration-debate>. [Consulta 23-10-2007].

PNUD. 1994. Informe sobre el Desarrollo Humano. México, D.F.: Fondo de Cultura Económica.

Rojas, Francisco y Moufida Gaucha. (eds). 2001. Seguridad Humana, Prevención de Conflictos y Paz. Santiago, Chile: FLACSO-UNESCO.

Rotschild, Emma. 1995. "What is Security?". Daedalus 124(3): 53-98.

Sartori, Giovanni. 1970. "Concept Misformation in Comparative Politics". The American Political Science Review. 64(4): 1033-1053.

SIPRI. 2006. SIPRI Yearbook: Armaments, Disarmament and International Security. Oxford, Reino Unido: Oxford University Press.

Schelling, Thomas. 1960. The Strategy of Conflict. Cambridge, Massachusetts: Harvard University Press.

Shaw, Martin. 1994. Global Society and International Relations: Sociological Concepts and Political Perspectives. Cambridge: Polity Press.

Sheehan, Michael. 2005. International Security: An Analytical Survey. Boulder: Lynne Rienner.

Snyder, Jack. 2003. "Imperial Temptations". The National Interest 71(1): 29-40.

Stern, Jessica. 2003. "Al Qaeda: The Protean Enemy". Foreign Affairs 82(4): 27-40.

Suhrke, Astri. 1999. "Human Security and the Interests of States". Security Dialogue. 30 (3): 265-276.

Tickner, J. Ann. 1995. "Re-visioning Security". En International Relations Theory Today, editado por K. Booth y S. Smith (eds). Oxford : Oxford University Press, 175-197.

The Economist. 2004. "Stepping Up the Pressure: The Conflict in Sudan's Darfur Region". The Economist, $<$ www.economist.com>

The Economist. 2007. "The International Criminal Court Approaches Darful". The Economist. <www. economist.com>

Thomas, Nicholas y William Tow. 2002. "The Utility of Human Security: Sovereignty and Humanitarian Intervention". Security Dialogue 33(2): 177-192.

Ullman, Richard. 1983. "Redefining Security". International Security 8 (Spring): 129-153.

Van Evera, Stephen. 2001. The Causes of War. Cornell: Cornell University Press.

Walt, Stephen. 1991. "The Renaissance of Security Studies". International Studies Quarterly 35(2): 211-239.

Weiss, Thomas, Tatiana Emmerj, Richard Louis y Jolly. 2005. UN Voices: The Struggle for Development and Social Justice. Bloomington, Indiana: Indiana University Press.

Wolfers, Arnold. 1952. "National Security as an Ambiguous Symbol". Political Science Quarterly 67(4): 481-502.

Wright, Quincy. 1942. A Study of War. Chicago, Illinois: University of Chicago Press. 
Yuen, Foong. Khong Yuen. 1992. Analogies of War. Princeton: Princeton University Press.

Yuen, Foong. 2001. "Human Security: A Shotgun Approach to Alleviating Human". Misery? Global Governance 7(3): 231-236.

Arturo Sotomayor es profesor e investigador de la División de Estudios Internacionales del Centro de Investigación y Docencia Económicas (CIDE). Es el actual coordinador (con Mónica Serrano) del Grupo de Estudios de Seguridad Nacional e Internacional en México. Sus áreas de especialización son seguridad internacional, relaciones cívico-militares de América Latina y los estudios de paz y conflicto internacional. Sus publicaciones han aparecido en revistas tales como el Journal of Latin American Politics and Society y en Foro Internacional. Es egresado de la licenciatura en relaciones internacionales por el Instituto Tecnológico Autónomo de México (ITAM) y doctor en ciencia política por la Universidad de Columbia, en Nueva York.

(arturo.sotomayor@cide.edu) 